\title{
UNIVERSITY OF WISCONSIN RADIOCARBON DATES XXVII
}

\section{CHRISTINE A. PRIOR ${ }^{l}$ and LORI F. PHELPS}

Center for Climatic Research, Institute for Environmental Studies, University of WisconsinMadison, 1225 West Dayton Street, Madison, Wisconsin 53706 USA

\section{INTRODUCTION}

Procedures and equipment used in the University of Wisconsin laboratory have been described previously (Bender, Bryson \& Baerreis 1965; Steventon \& Kutzbach 1986). Except as otherwise indicated, wood, charcoal and peat samples are pretreated with dilute $\mathrm{NaOH}-\mathrm{Na}_{4} \mathrm{P}_{2} \mathrm{O}_{7}$ and dilute $\mathrm{HCl}$ before conversion to counting gas methane; when noted, marls and lake cores are treated with acid only.

The dates reported are based on the 5568-year half-life of ${ }^{14} \mathrm{C}$. The standard deviation quoted includes only $1 \sigma$ of the counting statistics of background, sample and standard counts. Background methane is prepared from anthracite (standard methane from NBS oxalic acid). The activities of the dated samples for which $\delta^{13} \mathrm{C}$ values are listed and the standard methane have been corrected to correspond to a $\delta^{13} \mathrm{C}$ value of $-25 \%$.

Sample descriptions are based on information supplied by those who submitted samples.

\section{ACKNOWLEDGMENTS}

This research is supported by the National Science Foundation under Grant \#ATM89-02849 and by the College of Letters and Science, University of Wisconsin-Madison. We thank the UWMadison Chemistry Department and Isabel Treichel for use of their isotope ratio mass spectrometer. We also wish to thank Raymond L. Steventon.

\section{ARCHAEOLOGICAL SAMPLES}

\section{UNITED STATES}

Illinois

\section{Marlen Miller (11HA318) series}

Wood charcoal and nutshell collected 1988 from the Marlen Miller Site, Hancock County $\left(40^{\circ} 25^{\prime} \mathrm{N}, 91^{\circ} 00^{\prime} \mathrm{W}\right)$ by R. Wagner and submitted by L. Conrad, Western Illinois University Archaeological Research Laboratory. The samples provide radiometric dates for a ceramic assemblage dominated by Weaver Plain.

\section{WIS-2115.}

Sample from Feature 12, a Weaver Phase, flat-bottomed pit containing nut-processing tools, Weaver rim sherds and preserved floral remains.

${ }^{1}$ Present address: Department of Anthropology, Institute of Geophysics and Planetary Physics, University of California, Riverside, California 92512 USA 
WIS-2116.

$1620 \pm 60$

$\delta^{13} \mathrm{C}=-26.4 \%$

Sample from Feature 20, a vertical walled, flat-bottomed pit containing reconstructible Weaver Plain vessels, floral and faunal remains.

WIS-2117.

$1440 \pm 50$

$\delta^{13} \mathrm{C}=-25.9 \%$

Sample from Feature 25, a Weaver Phase basin-shaped pit feature superimposed by another Weaver Phase pit containing reconstructible sections of a noded, Weaver Plain vessel and preserved floral and faunal remains.

\section{Minnesota}

\section{Farley Village (21HU2) series}

Wood charcoal and nutshell collected 1989 from the Farley Village Site, Houston County $\left(43^{\circ} 39^{\prime} 42^{\prime \prime} \mathrm{N}, 91^{\circ} 42^{\prime} 12^{\prime \prime} \mathrm{W}\right)$ and submitted by J. Gallagher, Mississippi Valley Archaeological Center. The samples date a proto-historic Oneota occupation.

WIS-2173.

$200 \pm 50$

Sample from Feature 2.

$\delta^{13} C=-26.9 \%$

WIS-2174.

$465 \pm 50$

Sample from Feature 19.

$\delta^{13} \mathrm{C}=-27.2 \% 0$

\section{Nebraska}

\section{Upper Blue River Basin (25BU37) series}

Wood charcoal collected 1989 from the upper Blue River Basin, Butler County $\left(41^{\circ} 05^{\prime} \mathrm{N}\right.$, $97^{\circ} 10^{\prime} \mathrm{W}$ ) by J. Ludwickson and R. Bozell and submitted by R. Bozell, Nebraska State Historical Society. The samples provide an age and phase-level cultural affiliation for Central Plains tradition expansion into the upper Blue River Basin.

WIS-2146.

$\mathbf{7 7 0} \pm \mathbf{5 0}$

Sample from Feature 8908, remains of a northwest centerpost of house.

$\delta^{13} \mathrm{C}=-25.1 \%$

WIS-2147.

$675 \pm 50$

$\delta^{13} \mathrm{C}=-24.7 \%$

Sample from Feature 8904, a trash-filled pit extending 0-72 $\mathrm{cm}$ below the house floor.

WIS-2148.

$680 \pm 50$

Sample from Feature 8909, remains of a southwest centerpost of the house. $\delta^{13} C=-27.4 \%$ o

WIS-2149.

$695 \pm 50$

$\delta^{13} \mathrm{C}=-25.9 \%$

Sample from Feature 8921, a trash-filled pit extending 0-125 $\mathrm{cm}$ below the house floor. 


\section{Lower Platte River Basin (25CC228) series}

Wood charcoal collected 1989 from the lower Platte River Basin, Cass County $\left(41^{\circ} 01^{\prime} \mathrm{N}, 96^{\circ} 18^{\prime} \mathrm{W}\right)$ by J. Ludwickson and R. Bozell and submitted by R. Bozell. The dates add to the chronology of Nebraska Phase adaptations in the lower Platte River Basin (Blakeslee \& Caldwell 1979).

WIS-2150.

$660 \pm 50$

$\delta^{13} \mathrm{C}=-25.6 \%$

Sample from Feature 8904, a trash-filled pit extending 0-65 $\mathrm{cm}$ below the floor.

WIS-2151.

$595 \pm 50$

Sample probably from a wall post or roof rafter.

$\delta^{13} \mathrm{C}=-26.1 \%$ 。

\section{Platte and Loup River Basin Uplands (25HL28) series}

Wood charcoal collected 1985 from this site, Hall County $\left(40^{\circ} 55^{\prime} \mathrm{N}, 98^{\circ} 41^{\prime} \mathrm{W}\right)$ by W. Hunt and submitted by R. Bozell. The dates help determine upland settlement ages.

WIS-2154.

Sample probably from a roof rafter or wall post remnant.

WIS-2155.

Sample probably from a roof rafter or wall post remnant.
$870 \pm 40$

$\delta^{13} \mathrm{C}=-24.8 \%$

$830 \pm 50$

$\delta^{13} \mathrm{C}=-26.0 \% 0$

\section{Lower Platte River Basin (25SY31) series}

Wood charcoal collected 1984 from this site, Sarpy County $\left(41^{\circ} 2^{\prime} \mathrm{N}, 96^{\circ} 15^{\prime} \mathrm{W}\right)$ by J. Ludwickson and R. Bozell and submitted by R. Bozell. The dates help to separate chronologically the upper from the lower component at this site, and to refine the timing of the Nebraska Phase occupation of the lower Platte Basin (Blakeslee \& Caldwell 1979).

WIS-2152.

Sample from an activity area above a large Nebraska Phase lodge.

$\delta^{13} \mathrm{C}=-25.4 \%$

WIS-2153.

$930 \pm 50$

Sample from the floor of a house.

$\delta^{13} \mathrm{C}=-24.7 \%$

Ohio

\section{Petersen (330T9) series}

Wood charcoal collected 1989 from the Petersen Site $\left(42^{\circ} 30^{\prime} 23^{\prime \prime} \mathrm{N}, 83^{\circ} 1^{\prime} 15^{\prime \prime} \mathrm{W}\right)$ and submitted by D. Stothers, University of Toledo, Ohio. Dates help determine Indian Hills and Wolf Phases (Stothers \& Pratt 1980; Stothers \& Graves 1985; Graves 1984; Stothers \& Abel 1989).

WIS-2133.

$210 \pm 50$

Sample from Feature 2, a pit $30-70 \mathrm{~cm}$ below surface.

$\delta^{13} \mathrm{C}=-26.3 \%$ 
WIS-2134.

$$
\begin{array}{r}
\mathbf{3 8 0} \pm \mathbf{5 0} \\
\delta^{13} C=-26.7 \% 0 \\
\mathbf{2 4 0} \pm \mathbf{4 0} \\
\delta^{13} C=-26.2 \% 0
\end{array}
$$

Sample from Feature 34, a pit $25-40 \mathrm{~cm}$ below surface.

WIS-2135

Sample from Feature 49 , a pit $30-40 \mathrm{~cm}$ below surface.

Wisconsin

\section{Statz (47DA642) series}

Wood charcoal collected 1990 from the Statz Site, Dane County $\left(43^{\circ} 12^{\prime} \mathrm{N}, 89^{\circ} 29^{\prime} \mathrm{W}\right)$ by P. Coutch, P. Ladwig, M. Schlafmann, P. Nepokroeff and S. Brown, and submitted by N. Meinholz and S. Brown, State Historical Society, Madison. The sample was recovered from a semisubterranean structure to date the use of the structure.

WIS-2205.

Sample from Feature 90.

WIS-2206.

Sample from Feature 90.

WIS-2207.

Sample from Feature 128.

WIS-2210.

Sample from Feature 134.

WIS-2211.

Sample from Feature 144.

$$
\begin{array}{r}
1060 \pm \mathbf{5 0} \\
\delta^{13} C=-26.6 \% 0 \\
1010 \pm \mathbf{5 0} \\
\delta^{13} C=-26.3 \% 0 \\
840 \pm \mathbf{5 0} \\
\delta^{13} C=-26.4 \% 0 \\
1040 \pm \mathbf{5 0} \\
\delta^{13} C=-26.7 \% 0 \\
\mathbf{8 9 0} \pm \mathbf{5 0} \\
\delta^{13} C=-27.0 \% 0
\end{array}
$$

\section{Pike River series}

Wood charcoal collected 1990 from the Pike River Site, Kenosha County $\left(42^{\circ} 38^{\prime} \mathrm{N}, 87^{\circ} 49^{\prime} \mathrm{W}\right)$ by D. Wasion and submitted by D. Joyce, Kenosha Public Museum. The samples help date the Middle to Late Woodland transition.

WIS-2229. $910 \pm 50$

Sample from $11-12 \mathrm{~cm}$ below surface. $\delta^{13} \mathrm{C}=-26.8 \%$ 。

WIS-2230.

$$
1350 \pm 50
$$

Sample from Feature 14, a hearth.

$\delta^{13} \mathrm{C}=-27.5 \%$

Filer (47LC149) series

Wood charcoal collected 1988 and 1989 from the Filler Site, LaCrosse County (43.56 $30^{\prime \prime} \mathrm{N}$, $91^{\circ} 15^{\prime} 20^{\prime \prime} \mathrm{W}$ ) by J. Paulson, J. Vradenburg, M. O’Malley and P. Becker, and submitted by J. T. 
Penman, State Historical Society, Madison. Dates from this site determined that this Oneota occupation was not contemporary to the adjacent Ot Site (Penman 1984) (see below).

WIS-2120.

$270 \pm 50$

Sample from Feature 1.

$\delta^{13} \mathrm{C}=-26.3 \%$

WIS-2121.

$220 \pm 50$

Sample from Feature 2.

$\delta^{13} \mathrm{C}=-25.4 \%$

WIS-2122.

$230 \pm 60$

Sample from Feature 3.

$\delta^{13} C=-26.3 \%$

WIS-2194.

$\mathbf{3 7 0} \pm \mathbf{5 0}$

Sample from Feature 61.

$\delta^{13} \mathrm{C}=-27.1 \%$

WIS-2201.

$345 \pm 55$

Sample from Feature 16.

$\delta^{13} C=-25.6 \%$

WIS-2202.

$260 \pm 50$

Sample from Feature 36.

$\delta^{13} \mathrm{C}=-25.6 \%$

WIS-2203.

$310 \pm 50$

Sample from Feature 47.

$\delta^{13} \mathrm{C}=-25.9 \%$

WIS-2204.

$260 \pm 50$

Sample from Feature 63.

$\delta^{13} \mathrm{C}=-26.3 \%$ 。

\section{WIS-2114. Firesign (47LC359)}

$460 \pm 50$

$\delta^{13} \mathrm{C}=-25.9 \%$

Wood charcoal collected 1988 from the Firesign Site, LaCrosse County $\left(44^{\circ} 56^{\prime} \mathrm{N}, 91^{\circ} 16^{\prime} \mathrm{W}\right)$ by $\mathrm{M}$. O'Malley and submitted by J. T. Penman. This sample dates the latest prehistoric occupation at the Tremaine village complex.

\section{Midway Village (47LC19) series}

Wood charcoal collected 1984, 1985, and 1988 from the Midway Village Site, LaCrosse County $\left(43^{\circ} 56^{\prime} 35^{\prime \prime} \mathrm{N}, 91^{\circ} 16^{\prime} 05^{\prime \prime} \mathrm{W}\right)$ by R. Boszhardt, J. Gallagher, R. Rodell and A. LaFond, and submitted by R. Boszhardt, Mississippi Valley Archaeological Center. Oneota vessel pieces were also found at the site.

WIS-2107.

$300 \pm 50$

Sample from Feature 2.

$\delta^{13} \mathrm{C}=-26.2 \%$

WIS-2108.

$$
650 \pm 50
$$

Sample from Feature 89, a pit.

$\delta^{13} \mathrm{C}=-26.2 \%$ 。 
WIS-2109.

$470 \pm 50$

Sample from Feature 222.

$\delta^{13} \mathrm{C}=-26.1 \%$

WIS-2110.

$440 \pm 50$

Sample from Feature 234, a refuse pit.

$\delta^{13} C=-26.9 \%$

WIS-2111.

$610 \pm 50$

Sample from Feature 276, a pit.

$\delta^{13} \mathrm{C}=-26.0 \%$

WIS-2112.

$570 \pm 50$

Sample from Feature 2, a storage pit.

$\delta^{13} \mathrm{C}=-21.1 \%$ 。

\section{Ot (47LC262) series}

Wood charcoal collected 1988 and 1989 from the Ot Site, LaCrosse County $\left(43^{\circ} 56^{\prime} 30^{\prime \prime} \mathrm{N}\right.$, $\left.91^{\circ} 15^{\prime} 30^{\prime \prime} \mathrm{W}\right)$ by J. O'Gorman, A. Olson, P. Becker and J. Evanson, and submitted by J. T. Penman. A cemetery at this site was dated previously (Broihahn, Penman \& Rusch 1987; Steventon \& Kutzbach 1988).

WIS-2118.

Sample from Feature 19.

WIS-2119.

Sample from Feature 30.

WIS-2184.

Sample from Feature 13, a trash pit.

WIS-2185.

Sample from Feature 31, a trash pit.

WIS-2186.

Sample from Feature 47.

WIS-2187.

Sample from Feature 62, a trash pit.

WIS-2188.

Sample from Feature 82.

WIS-2189.

Sample from Feature 93.
$260 \pm 70$

$\delta^{13} \mathrm{C}=-26.5 \%$

$510 \pm 50$

$\delta^{13} \mathrm{C}=-26.4 \%$ 。

$210 \pm 50$

$\delta^{13} \mathrm{C}=-26.0 \%$

$300 \pm 50$

$\delta^{13} C=-25.4 \%$

$415 \pm 50$

$\delta^{13} \mathrm{C}=-26.9 \%$

$270 \pm 50$

$\delta^{13} \mathrm{C}=-25.7 \%$

$310 \pm 50$

$\delta^{13} \mathrm{C}=-26.1 \%$

$390 \pm 50$ 
WIS-2190.

Sample from Feature 136.

WIS-2192.

Sample from Feature 163.

WIS-2192.

Sample from Feature 172.

WIS-2193.

Sample from Feature 183.

$$
\begin{array}{r}
\mathbf{3 4 0} \pm \mathbf{5 0} \\
\delta^{13} C=-26.3 \% 0 \\
\mathbf{3 5 0} \pm \mathbf{5 0} \\
\delta^{13} C=-25.4 \% 0 \\
\mathbf{2 2 0} \pm \mathbf{5 0} \\
\delta^{13} C=-25.5 \% 0 \\
\mathbf{4 6 0} \pm \mathbf{5 0} \\
\delta^{13} C=-26.3 \% 0
\end{array}
$$

\section{Pammel Creek (47LC61) series}

Wood charcoal collected 1989 at the Pammel Creek Site, LaCrosse County $\left(43^{\circ} 45^{\prime} 44^{\prime \prime} \mathrm{N}\right.$, $91^{\circ} 12^{\prime} 35^{\prime \prime} \mathrm{W}$ ) and submitted by C. Arzigian, Mississippi Valley Archaeological Center. These samples help date Oneota cultural artifacts and the transitional period between the Brice Prairie Phase and the Valley View Phase.

WIS-2160

$530 \pm 50$

Sample from Feature 137, a medium-sized basin, $60 \mathrm{~cm}$ deep.

$\delta^{13} \mathrm{C}=-26.3 \%$

WIS-2162.

$470 \pm 50$

Sample from Feature 139, a basin, $90 \mathrm{~cm}$ deep.

$\delta^{13} \mathrm{C}=-26.2 \%$

WIS-2162

$445 \pm 50$

Sample from Feature 143, a pit, $60 \mathrm{~cm}$ deep.

$\delta^{13} C=-26.6 \%$

WIS-2163.

$570 \pm 50$

Sample from Feature 144, a basin, $20 \mathrm{~cm}$ deep.

$\delta^{13} \mathrm{C}=-25.8 \%$

WIS-2164.

$380 \pm 50$

Sample from Feature 156, a pit, $60 \mathrm{~cm}$ deep.

$\delta^{13} C=-26.6 \% \circ$

\section{Tremaine (47LC95) series}

Wood charcoal collected 1988 from the Tremaine Site, LaCrosse County $\left(43^{\circ} 56^{\prime} 48^{\prime \prime} \mathrm{N}, 91^{\circ} 15^{\prime} 50^{\prime \prime} \mathrm{W}\right)$ by P. Becker, K. Kachel, J. Vradenburg and M. O'Malley, and submitted by J. T. Penman. These samples help date an Oneota occupation.

WIS-2123.

Sample from Feature 11.

$\delta^{13} C=-26.9 \% \circ$

WIS-2124.

$1480 \pm 50$

Sample from Feature 17.

$\delta^{13} \mathrm{C}=-25.8 \%$ 。 
WIS-2125.

$450 \pm 40$

Sample from Feature 20.

$\delta^{13} C=-26.6 \% \circ$

WIS-2126.

$600 \pm 50$

Sample from Feature 32.

$\delta^{13} \mathrm{C}=-26.7 \%$

WIS-2195.

$720 \pm 50$

Sample from Feature 17.

$\delta^{13} \mathrm{C}=-25.8 \%$

$\mathbf{7 7 0} \pm \mathbf{5 0}$

WIS-2113. Cabbage Patch (470U103)

$\delta^{13} \mathrm{C}=-25.7 \%$

Wood charcoal collected 1988 from the Cabbage Patch Site, Outagamie County by B. Workmaster and C. Abrams, and submitted by L. Rusch, Wisconsin State Historical Society. This feature contained Late Woodland pottery types, Point Sauble collared and Madison plain. The sample dates a Late Woodland occupation (Rusch 1988).

\section{Hulburt Creek series}

Wood charcoal and charred seeds collected 1990 from the Hulburt Creek Site, Sauk County $\left(43^{\circ} 37^{\prime} \mathrm{N}, 89^{\circ} 50^{\prime} \mathrm{W}\right)$ and submitted by W. Gartner, Geography Department, University of Wisconsin-Madison. These samples will help date the construction of ditched field agriculture.

WIS-2214.

$980 \pm 50$

Sample from $23-27 \mathrm{~cm}$ below the planting surface.

$\delta^{13} \mathrm{C}=-23.0 \%$

WIS-2215.

$920 \pm 50$

Sample from $10-40 \mathrm{~cm}$ below the planting surface.

$\delta^{13} \mathrm{C}=-24.2 \%$

\section{Viola Rockshelter (47VE640) series}

Wood charcoal collected 1980 and 1986 from the Viola Rockshelter Site, Vernon County $\left(43^{\circ} 28^{\prime} 38^{\prime \prime} \mathrm{N}, 90^{\circ} 41^{\prime} 15^{\prime \prime} \mathrm{W}\right)$ and submitted by J. Theler, Mississippi Valley Archaeological Center. Ceramics were recovered from the site.

WIS-2105.

$1220 \pm 70$

Sample from Feature 3.

$\delta^{13} \mathrm{C}=-26.5 \%$

WIS-2106.

$1810 \pm 80$

Sample from a test pit.

$\delta^{13} C=-26.3 \% \circ$

\section{CHILE}

WIS-2208. San Miguel

$450 \pm 50$

$\delta^{13} \mathrm{C}=-23.3 \%$ o

Charcoal ash collected 1990 from San Miguel, Ovalle-Valle Limari, IV Region, Chile $\left(30^{\circ} 0^{\prime} \mathrm{S}\right.$, 
$71^{\circ} 0^{\prime} \mathrm{W}$ ) by M. Biskupovic and submitted by M. Rosado, Rutgers University. The sample corresponds to a settlement site of the Diaguita Classical period.

ECUADOR

WIS-2131. Hacienda Pirincay

$2080 \pm 60$

$\delta^{13} \mathrm{C}=-25.3 \%$

Charcoal collected 1988 from Hacienda Pirincay, Canton Paute, Province of Azuay $\left(2^{\circ} 35^{\prime}\right.$ S, $78^{\circ} 44^{\prime} \mathrm{W}$ ) and submitted by K. Olsen Bruhns, San Francisco State University. The sample should date the leveling of the site's center and construction of a large pavement, complete with stonebuilt drains.

PAKISTAN

\section{Harappa series}

Wood charcoal collected 1988 and 1989 from Harappa, District Sahiwal, Punjab (30 38' N, $72^{\circ} 52^{\prime} \mathrm{E}$ ) and submitted by J. M. Kenoyer, Anthropology Department, University of WisconsinMadison. These dates provide a chronological sequence for the expansion of a proto-urban and subsequent urban settlement at Harappa, Pakistan.

WIS-2139.

Sample from Lot 360, a hearth.

WIS-2140.

Sample from Lot 556.

WIS-2141.

Sample from Lot 772.

WIS-2142

Sample from Lot 722 , Mound E.

WIS-2143.

Sample from Lot 798, a hearth.

WIS-2144.

Sample from Lot 529.

WIS-2145

Sample from Lot 738.
$3820 \pm 60$

$\delta^{13} C=-25.2 \%$ 。

$4290 \pm 70$

$\delta^{13} C=-23.4 \%$

$3920 \pm 70$

$\delta^{13} \mathrm{C}=-23.2 \%$

$4135 \pm 65$

$\delta^{13} C=-25.6 \%$

$3825 \pm 60$

$\delta^{13} \mathrm{C}=-26.7 \%$

$3720 \pm 100$

$\delta^{13} \mathrm{C}=-26.4 \%$

$4020 \pm 60$

$\delta^{13} \mathrm{C}=-25.9 \%$ 


\section{GEOLOGICAL SAMPLES}

UNITED STATES

Massachusetts

\section{Great Pond series}

A core collected 1989 from Great Pond, Wellfleet, Barnstable County $\left(41^{\circ} 56^{\prime} 25^{\prime \prime} \mathrm{N}, 70^{\circ} 00^{\prime} 03^{\prime \prime} \mathrm{W}\right)$ and submitted by M. Winkler, Center for Climatic Research, University of Wisconsin-Madison.

WIS-2129

$1025 \pm 50$

Gyttja, 0-10 cm depth.

$\delta^{13} \mathrm{C}=-25.9 \%$

WIS-2130

$2120 \pm 60$

Gyttja, 68-79 cm depth.

$\delta^{13} \mathrm{C}=-26.0 \%$

WIS-2169. Gull Pond

$5165 \pm 60$

$\delta^{13} \mathrm{C}=-24.4 \%$

Gyttja, from $72-78 \mathrm{~cm}$ below the sediment/water interface was collected from a core October 1989 from Gull Pond, Barnstable County $\left(41^{\circ} 57^{\prime} 17^{\prime \prime} \mathrm{N}, 70^{\circ} 00^{\prime} 32^{\prime \prime} \mathrm{W}\right)$ by M. Winkler, P. Sanford, K. Hobler, R. Webb, P. Johanson and D. Manski, and submitted by M. Winkler. This date will be used in describing the evolution of Gull Pond. The sample received acid treatment only.

\section{WIS-2172. Herring Pond}

$2460 \pm 60$

Gyttja, from 171-176 cm below the sediment/water interface was collected with a Livingstone corer October 1989 from Herring Pond, Barnstable County $\left(41^{\circ} 57^{\prime} 46^{\prime \prime} \mathrm{N}, 70^{\circ} 00^{\prime} 49^{\prime \prime} \mathrm{W}\right)$ by $\mathrm{M}$. Winkler $e t$ al. and submitted by M. Winkler. The sample helps provide a chronology for the development of the Gull Pond chain of ponds.

\section{WIS-2171. Higgins Pond}

$2150 \pm 60$

$\delta^{13} C=-29.2 \%$

Gyttja, from $200-206 \mathrm{~cm}$ below the sediment/water interface was collected with a Livingstone corer October 1989 from Higgins Pond, Barnstable County $\left(41^{\circ} 57^{\prime} 51^{\prime \prime} \mathrm{N}, 70^{\circ} 00^{\prime} 32^{\prime \prime} \mathrm{W}\right)$ by $\mathrm{M}$. Winkler et al. and submitted by M. Winkler. The sample helps extend the chronology for the Gull Pond chain.

\section{WIS-2170. Williams Pond}

$9500 \pm 90$

$$
\delta^{13} \mathrm{C}=-27.1 \% \text { 。 }
$$

Gyttja, from 74-82.5 cm depth was collected with a Livingstone corer October 1989 from Williams Pond, Barnstable County $\left(41^{\circ} 57^{\prime} 49^{\prime \prime} \mathrm{N}, 70^{\circ} 00^{\prime} 30^{\prime \prime} \mathrm{W}\right)$ by M. Winkler et al. and submitted by $\mathrm{M}$. Winkler. The sample dates the beginning of lake sediment deposition during the LateGlacial/Holocene in Williams Pond. 


\section{Minnesota}

\section{Elk Lake series}

A core collected 1989 from Elk Lake, Grant County $\left(45^{\circ} 52^{\prime} \mathrm{N}, 95^{\circ} 48^{\prime} \mathrm{W}\right)$ and submitted by A. Smith, Brown University. These dates, along with pollen analysis, identified a hiatus as midHolocene.

WIS-2165

$6470 \pm 50$

Sample from $1136-1140 \mathrm{~cm}$ below surface.

$\delta^{13} \mathrm{C}=-17.0 \%$

WIS-2166

$6860 \pm 70$

Sample from $1126-1132 \mathrm{~cm}$ below surface.

$\delta^{13} C=-14.5 \%$ o

WIS-2167.

$6240 \pm 70$

Sample from $1010-1027 \mathrm{~cm}$ below surface.

$\delta^{13} \mathrm{C}=-15.1 \%$

WIS-2168.

$3590 \pm 150$

Sample from $883-893 \mathrm{~cm}$ below surface.

$\delta^{13} \mathrm{C}=-19.6 \%$

\section{Montana}

WIS-2176. Upper Red Rock Lake

$\mathbf{4 2 , 8 0 0} \pm \mathbf{3 4 5 0}$

$\delta^{13} \mathrm{C}=-11.5 \%$ 。

A core collected 1989 from Upper Red Rock Lake, Beaverhead County $\left(44^{\circ} 36^{\prime} \mathrm{N}, 111^{\circ} 43^{\prime} \mathrm{W}\right)$ and submitted by C. Whitlock, Geography Department, University of Oregon. The sample was from 7.12-7.44 $\mathrm{m}$ below the water surface.

\section{Pintlar Lake series}

A core collected 1989 from Pintlar Lake, Deer Lodge County $\left(45^{\circ} 50^{\prime} \mathrm{N}, 113^{\circ} 40^{\prime} \mathrm{W}\right)$ and submitted by C. Whitlock.

WIS-2175.

Gyttja, $6.5-6.6 \mathrm{~m}$ below the water surface.

WIS-2177.

Gyttja, 8.1-8.2 m depth.

WIS-2178.

Gyttja, 9.5-9.6 m depth.

WIS-2179.

Gyttja, $11.06-11.18 \mathrm{~m}$ depth.
$1795 \pm 50$

$\delta^{13} \mathrm{C}=-27.8 \%$ 。

$2130 \pm 160$

$\delta^{13} \mathrm{C}=-27.4 \% \circ$

$4120 \pm 60$

$\delta^{13} C=-27.6 \% \circ$

$$
5830 \pm 70
$$

$\delta^{13} \mathrm{C}=-27.2 \%$ 
WIS-2180.

Gyttja, $12.9-13.0 \mathrm{~m}$ depth.

WIS-2181.

$9860 \pm 90$

Gyttja, 13.5-13.6 m depth.

$\delta^{13} \mathrm{C}=-27.4 \%$ 。

WIS-2182.

$10,690 \pm 100$

Gyttja, 13.7-13.8 m depth.

$\delta^{13} C=-27.4 \% 0$

\section{South Dakota}

\section{WIS-2213. Ortonville Stone Quarry}

$\mathbf{4 2 , 4 6 0 \pm 4 0 0 0}$

$\delta^{13} \mathrm{C}=-26.2 \% 0$

Wood collected 1989 from Ortonville Quarry, Lac Qui Parle County $\left(45^{\circ} 16^{\prime} \mathrm{N}, 96^{\circ} 24^{\prime} \mathrm{W}\right)$ by J. Gilbertson and M. Bratrud, and submitted by J. Gilbertson, South Dakota Geological Society. The date confirms a pre-Late Wisconsin age for overlying till deposited by the glacial River Warren.

Wisconsin

WIS-2159. Long Island

$210 \pm 50$

$\delta^{13} \mathrm{C}=-26.8 \%$ o

Fibric peat collected 1989 at Long Island, Ashland County $\left(46^{\circ} 43^{\prime} \mathrm{N}, 90^{\circ} 46^{\prime} \mathrm{W}\right)$ by D. Kiesel and submitted by L. Bona, Geology Department, University of Wisconsin-Madison. The sample was from 115-125 cm depth and should give a minimum age of foreshore deposits in the beach ridge (Swain 1981).

\section{Otter Island series}

Decomposed sphagnum peat collected 1989 at Otter Island, Apostle National Lakeshore, Ashland County and submitted by A. Swain, Center for Climatic Research, University of WisconsinMadison. The samples date the beginning of a decrease of pine and increase of birch, spruce and hemlock in the area.

WIS-2127.

Peat, $50-55 \mathrm{~cm}$ below the present peat surface.

WIS-2128.

Peat, $75-80 \mathrm{~cm}$ below the present peat surface.

$$
\begin{array}{r}
\mathbf{2 4 6 0} \pm \mathbf{6 0} \\
\delta^{13} C=-27.4 \% 0 \\
\mathbf{3 8 9 0} \pm \mathbf{7 0} \\
\delta^{13} C=-28.1 \% 0
\end{array}
$$

\section{Chub Lake series}

A core collected 1988 from Chub Lake, Dodge County $\left(43^{\circ} 13^{\prime} \mathrm{N}, 88^{\circ} 53^{\prime} \mathrm{W}\right)$ and submitted by $\mathrm{M}$. Kolb, Department of Geosciences, University of Wisconsin-Milwaukee. These dates will determine the chronology of Holocene lacustrine deposition in southeastern Wisconsin. 
WIS-2222.

$13,250 \pm 130$

Silty clay, $2 \mathrm{~m}$ below the sediment surface.

$\delta^{13} \mathrm{C}=-22.0 \%$

WIS-2223.

$12,720 \pm 130$

Silty clay, $3 \mathrm{~m}$ below the sediment surface.

$\delta^{13} \mathrm{C}=-20.0 \%$

WIS-2224.

$14,450 \pm 145$

Silty clay, $6 \mathrm{~m}$ below the sediment surface.

$\delta^{13} \mathrm{C}=-18.4 \%$

\section{WIS-2132. Hinderman}

$1270 \pm 50$

$\delta^{13} \mathrm{C}=-25.8 \%$

Wood collected 1988 at the Hinderman Site, Grant County $\left(42^{\circ} 39^{\prime} \mathrm{N}, 90^{\circ} 35^{\prime} \mathrm{W}\right)$ by J. C. Knox and D. S. Leigh, and submitted by J. C. Knox, University of Wisconsin-Madison. The date records the slow natural rate of floodplain vertical accretion vs. human-accelerated accretion (Knox 1985, 1987).

\section{WIS-2209. Pike River}

$10,320 \pm 100$

$\delta^{13} \mathrm{C}=-26.7 \% 0$

A wood fragment collected 1990 at Pike River Dike Retention Basin, Richland County $\left(43^{\circ} 22^{\prime} 48^{\prime \prime} \mathrm{N}, 90^{\circ} 21^{\prime} 41^{\prime \prime} \mathrm{W}\right)$ by P. Cahfer and M. Foreman, and submitted by D. Omernik, USDA Soil Conservation Service. The date is significant in determining the amount of deposition and soil development processes occurring after deposition.

PERU

\section{Laguna Jeronimo series}

Lake sediment collected 1987 at Laguna Jeronimo, central Peru $\left(11^{\circ} 47^{\prime} \mathrm{S}, 75^{\circ} 13^{\prime} \mathrm{W}\right)$ and submitted by $\mathrm{H}$. E. Wright, Limnological Research, University of Minnesota, Minneapolis. The objective is to date the end of Late-Pleistocene glaciation in the Peruvian Andes (Wright, Seltzer \& Hanson 1989.)

WIS-2156.

Organic lake sediment, $895-902.5 \mathrm{~cm}$ below the water surface.

WIS-2157.

Organic lake sediment, $997.5-1002.5 \mathrm{~cm}$ below the water surface.

\section{WIS-2158. Laguna Pomacocha}

Organic lake sediment collected 1987 at Laguna Pomacocha, central Peruvian Andes $\left(11^{\circ} 46^{\prime} \mathrm{S}\right.$, $75^{\circ} 16^{\prime} \mathrm{W}$ ) and submitted by $\mathrm{H}$. E. Wright. The sample is from $710-715 \mathrm{~cm}$ below the water surface, and is used to date pollen stratigraphy in the Holocene (Wright, Seltzer \& Hanson 1989). 


\section{REFERENCES}

Bender, M. M., Bryson, R. A. and Baerreis, D. A. 1965 University of Wisconsin radiocarbon dates I. Radiocarbon 7: 399-407.

Blakeslee, D. and Caldwell, W. 1979 The Nebraska Phase: An Appraisal. Reprints in Anthropology 18. Lincoln, Nebraska, J\&L Reprint Company.

Broihahn, J. H., Penman, J. T. and Rusch, L. 1987 Transportation archaeology in Wisconsin: The 1986 field season. Wisconsin Department of Transportation Archaeology Report 13.

Graves, J. (ms.) 1984 The Indian Hills site (33WO4). Archaeological reflections of a protohistoric assistaeronon town. M.A. thesis, University of Toledo, Ohio.

Knox, J. C. 1985 Responses of floods to Holocene climatic change in the Upper Mississippi Valley. Quaternary Research 23: 287-300.

1987 Historical valley floor sedimentation in the upper Mississippi Valley. Annals of the Association of American Geographers 77: 224-244.

Penman, J. T. 1984 Archaeology of the Great River Road: Summary report. Wisconsin Department of Transportation, Archaeology Report 10.

Rusch, L. A. (ms.) 1988 Archaeological test excavations at the Dowser (OU100), Tews (OU102), Cabbage
Patch (OU103), and Cleo's (OU104) sites, Outagamie County, Wisconsin. Report on file at the State Historic Preservation Office \& State Historical Society of Wisconsin Museum.

Steventon, R. L. and Kutzbach, J. E. 1986 University of Wisconsin radiocarbon dates XXIII. Radiocarbon 28(3) 1206-1223.

1988 University of Wisconsin radiocarbon dates XXV: Radiocarbon 30(3): 367-383.

Stothers, D. and Abel, T. 1989 The position of the "Pearson complex" in the late prehistory of northern Ohio. Archaeology of Eastern North America: 192 p. Stothers, D. and Graves, J. 1985 The Prairie Peninsula co-tradition. Archaeology of Eastern North America 13: $153-175$

Stothers, D. and Pratt, G. 1980 Cultural continuity and change in the region of the Western Lake Basin. Toledo Area Aboriginal Research Bulletin 9: 1-38.

Swain, A. (ms.) 1981 Final report to the national park service on forest and disturbance history of Apostle Islands. Center for Climatic Research, University of Wisconsin, Madison.

Wright, H. E., Seltzer, G. and Hanson, B. 1989 Glacial and climatic history of the central Peruvian Andes. National Geographic Research 5: 439-446. 